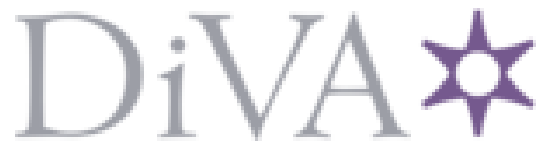

http://www.diva-portal.org

Preprint

This is the submitted version of a paper published in Solid-State Electronics.

Citation for the original published paper (version of record):

Ayala, C L., Grogg, D., Bazigos, A., Bleiker, S J., Fernandez-Bolanos, M. et al. (2015)

Nanoelectromechanical digital logic circuits using curved cantilever switches with amorphous-carbon-coated contacts

Solid-State Electronics, 113: 157-166

https://doi.org/10.1016/j.sse.2015.05.029

Access to the published version may require subscription.

N.B. When citing this work, cite the original published paper.

Permanent link to this version:

http://urn.kb.se/resolve?urn=urn:nbn:se:kth:diva-173125 


\title{
Nanoelectromechanical Digital Logic Circuits Using Curved Cantilever Switches with Amorphous-Carbon-Coated Contacts
}

\author{
Christopher L. Ayala ${ }^{\mathrm{a}, *}$, Daniel Grogg ${ }^{\mathrm{b}}$, Antonios Bazigos $^{\mathrm{c}}$, Simon J. Bleiker ${ }^{\mathrm{d}}$, Montserrat Fernandez-Bolaños ${ }^{\mathrm{c}}$, \\ Frank Niklaus ${ }^{\mathrm{d}}$, Christoph Hagleitner ${ }^{\mathrm{a}, *}$ \\ ${ }^{a}$ IBM Research - Zurich, CH-8803 Rüschlikon, Switzerland \\ ${ }^{b}$ now at TE Connectivity, $\mathrm{CH}-8804 \mathrm{Au}$, Switzerland \\ ${ }^{c}$ École Polytechnique Fédérale de Lausanne (EPFL), CH-1015 Lausanne, Switzerland \\ ${ }^{d}$ KTH Royal Institute of Technology, SE-100 44, Stockholm, Sweden
}

\begin{abstract}
Nanoelectromechanical (NEM) switches have the potential to complement or replace traditional CMOS transistors in the area of ultra-low-power digital electronics. This paper reports the demonstration of prototype circuits including the first 3-stage ring oscillator built using cell-level digital logic elements based on curved NEM switches. The ring oscillator core occupies an area of $30 \mu \mathrm{m} \times 10 \mu \mathrm{m}$ using $6 \mathrm{NEM}$ switches. Each NEM switch device has a footprint of $5 \mu \mathrm{m} \times 3 \mu \mathrm{m}$, an air gap of $60 \mathrm{~nm}$ and is coated with amorphous carbon (a-C) for reliable operation. The ring oscillator operates at a frequency of $6.7 \mathrm{MHz}$, and confirms the simulated inverter propagation delay of $25 \mathrm{~ns}$. The successful fabrication and measurement of this demonstrator are a key milestone on the way towards an optimized, scaled technology with sub-nanosecond switching times, lower operating voltages and VLSI implementation.
\end{abstract}

Keywords: NEMS, MEMS, ring oscillator, VLSI, digital logic design, curved cantilever, amorphous carbon.

\section{Introduction}

CMOS has been the predominant technology for digital integrated circuits since the semiconductor industry made the transition from NMOS technology in the early 1980s. Present-day microprocessors are manufactured using a $22 \mathrm{~nm}$ CMOS process with a transition to $14 \mathrm{~nm}$ technology starting at the end of 2014 [1, 2]. Continuing to shrink devices beyond the $8 \mathrm{~nm}$ technology node requires tremendous efforts in devices, materials and process research.

There are two fundamental issues that limit progress for ultra-low-power applications in CMOS:

1. The leakage current of circuits built using high-performance CMOS technologies is approaching the dynamic current. Only a few high-performance computing applications have circuit-activity levels that contain the leakage power at a reasonable level of $<10 \%$ of the active power. The power-density limitations $(\sim 100 \mathrm{~W}$ per processor chip for desktop consumer applications, significantly less for embedded systems) further exacerbate this challenge.

2. The theoretical minimum for the subthreshold slope of silicon-based MOS transistors is $60 \mathrm{mV} /$ decade, which leads to a minimum supply voltage of $>400 \mathrm{mV}$ for practical applications [3]. Among other issues,

\footnotetext{
*Corresponding authors, email: chris.ayala@ieee.org; hle@zurich.ibm.com
}

this limits the gains in power efficiency obtainable by moving to next-generation CMOS technology.

Beyond these fundamental issues, the cost of using the latest CMOS technology node has reached a point where only a few (very) high-volume applications can afford it.

Given the scaling limits of silicon-based CMOS technology, alternative devices have been proposed including, but not limited to, carbon/graphene nanostructures, molecular devices, quantum devices and photonics [1]. Of the various beyond-CMOS device candidates, the nanoelectromechanical (NEM) switch shows promise because it offers a comparably simple fabrication process with fewer masks and allows co-integration with CMOS via a back-end-ofline (BEOL) process. It also addresses the aforementioned fundamental problems because the NEM switch has virtually zero leakage, and the abrupt switching characteristic (no subthreshold slope limitation) potentially allows the use of lower supply voltages $[4,5]$.

Several research groups have been or are currently investigating the design and applications of NEM switches for logic applications, including but not limited to [6-19].

Our work involves the development of a NEM-switchbased VLSI framework that encompasses device optimization and fabrication, BEOL processing, logic design, and device-/circuit-modeling. As a first key demonstrator of our approach, we implemented a ring oscillator consisting of 3 inverting stages using in-plane curved cantilever NEM switches coated with amorphous carbon (a-C) on the contact. The in-plane design is the basis to achieve the de- 


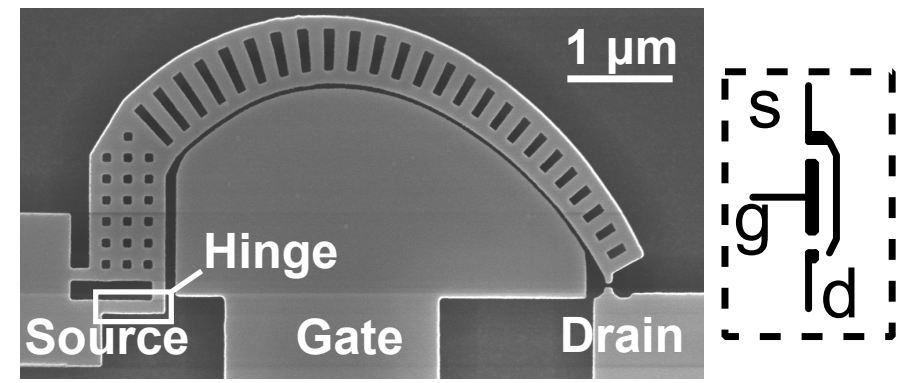

Figure 1: SEM image of a fabricated curved NEM switch with a hinge length of $0.5 \mu \mathrm{m}$ (stiff design) and corresponding circuit symbol on the right-hand side.

sign freedom required for curved cantilevers. It also solves many of the scalability issues that out-of-plane switches face, because the critical air gap is defined through a single vertical etching step. The switches have been developed for digital logic applications and feature a curved cantilever design for improved robustness over straight cantilevers $[20,21]$. Nanoscale conducting filaments are formed within the a-C contact material via Joule heating through the application of a sufficiently high voltage without creating large adhesion forces.

The reduced adhesion forces between the two a-C-coated contacts allow control of the hysteresis of the switch and, together with the inherent stability of the material, improve reliability [21]. A dedicated output stage has been developed to safely interface the NEM switch circuits with the large capacitive load of the external measurement equipment. The experimental measurements have shown the successful operation of a standalone single-stage inverter, a buffer, and a 3-stage ring oscillator operating at a frequency of $6.7 \mathrm{MHz}$. The measurements are consistent with simulations from both finite-element analysis and analytical compact modeling of the curved NEM switch.

The remainder of this paper is structured as follows: Section 2 describes the general features of our 3 -terminal NEM switch and how it is fabricated. Section 3 discusses our approach to bringing our devices to the level of VLSI implementation, including the BEOL integration process, standard cell design, interface circuits, and device/circuit modeling. Section 4 details prototype circuits we have fabricated and measured. We also show how well our measurement results align with our simulations. Finally, we conclude the paper in Section 5 .

\section{NEM Switch Device}

\subsection{Device Characteristics}

As shown in Figure 1, the NEM switch is designed using an in-plane curved cantilever approach, which provides a compact footprint and increased reliability . It consists of the following three terminals:

- Source: A fixed terminal and supporting anchor for an electrically equivalent, movable cantilever.
Step 1: E-beam lithography and ICP etching

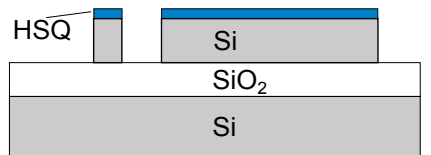

Step 3: Pt-Au metal evaporation

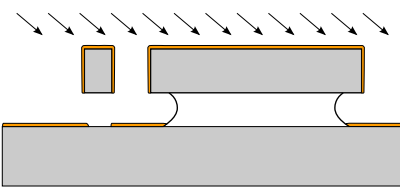

Step 2: BHF release and super critical point drying

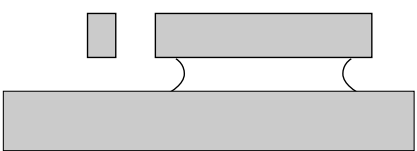

Step 4: a-C sputter deposition
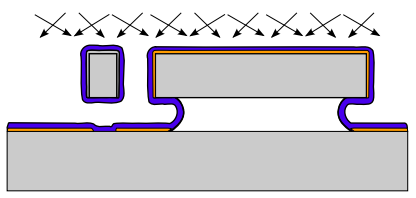

Figure 2: Fabrication process steps for the curved NEM switch.

- Gate: A fixed terminal creating an attracting electrical field to pull in the cantilever when there is a sufficient voltage difference between the source and gate. This voltage difference is defined as the pull-in voltage $\left(V_{\mathrm{pi}}\right)$.

- Drain: A fixed terminal where the cantilever tip makes contact, forming a connection between source and drain when the switch is closed.

The curved design mitigates the excessive field strength towards the edge of the cantilever, typically found in straight cantilever designs $[6,20,21]$. This improves the margin between $V_{\mathrm{pi}}$ and the breakdown voltage, thus increasing robustness. The length of the switch is $5 \mu \mathrm{m}$, with a $60 \mathrm{~nm}$ air gap between the actuating gate electrode and the cantilever in the open state. When the switch closes, this air gap is approximately half of the initial value. The design is scalable for smaller feature sizes, where a decreased air gap of $5 \mathrm{~nm}$ and a sub-micron device length enable subnanosecond switching times and operating voltages below $1 \mathrm{~V}[4,20]$.

The conduction coating consists of platinum with gold to reduce structural stress and eliminate out-of-plane bending of the switch. A thin layer of amorphous carbon (a$\mathrm{C}$ ) is also deposited onto the conduction layer to benefit from the lower surface energy [21]. Nanoscale contacts are formed in the a-C by applying a sufficiently high voltage in the range of $1.5 \mathrm{~V}$ to $2.5 \mathrm{~V}$, allowing current to flow through local filaments without large adhesive forces. This reduces the restoring force of the relay to less than $100 \mathrm{nN}$ and decreases the on-off hysteresis window from over $2 \mathrm{~V}$ for a platinum-platinum contact to $0.5 \mathrm{~V}$ for the a-C contact [21]. Reliability measurements show that the curved design combined with a-C can operate for over 100 million hot-switching cycles as a standalone switch [21]. After a sufficient number of conduction filaments in the a-C have been formed through repeated hot-switching, the onresistance $\left(R_{\text {on }}\right)$ drops to less than $50 \mathrm{k} \Omega$.

For the experiments presented in this paper, we used two NEM switches with different hinge lengths. The hinge defines the stiffness and, hence, the $V_{\mathrm{pi}}$ of the switch. 
Finite-element analyses have shown that a hinge of $0.5 \mu \mathrm{m}$ (stiff switch, Figure 1) corresponds to a $V_{\mathrm{pi}}$ of $10.3 \mathrm{~V}$ and an expected mechanical pull-in time of $23 \mathrm{ns,} \mathrm{whereas} \mathrm{a}$ hinge of $1.0 \mu \mathrm{m}$ (soft switch) corresponds to a $V_{\text {pi }}$ of $7.6 \mathrm{~V}$ and an expected mechanical pull-in time of $48 \mathrm{~ns}$.

\subsection{Device Fabrication}

The fabrication of the NEM switch (Figure 2) involves the use of electron beam lithography on a silicon-on-insulator (SOI) substrate combined with hydrogen-bromide-based inductively coupled plasma (ICP) etching of a 220-nmthick silicon device layer [21]. During this step, the 60-nm air gap is directly etched as well. Next, the silicon dioxide beneath the device layer is etched with an undercut, creating free-standing structures. The undercut eliminates the formation of electrical shortcuts through the conduction layer that is deposited in the next steps. To create the conduction layer, $2 \mathrm{~nm}$ of titanium, $10 \mathrm{~nm}$ of gold, and finally $20 \mathrm{~nm}$ of platinum are evaporated onto the structures. Lastly, a 10-nm layer of a-C is deposited in an argon atmosphere, optimized for low stress via sputter deposition. The simplicity of the deposition makes it suitable for full wafer integration.

\section{Towards NEM Switch VLSI Circuit Design}

To bring NEM switches into the realm of VLSI development, a BEOL integration process has been proposed to provide high-density interconnectivity between the NEM switch devices. Based on the BEOL framework, a standard approach to design logic cells has been developed to efficiently arrange switches in a manner suitable for complementary logic. In addition, peripheral circuits to safely interface and measure logic circuits have also been designed. Lastly, compact modeling of the NEM switch has been performed to conduct fast and reasonably accurate circuit simulations. These essential ingredients lay the foundation for VLSI circuit design using NEM switches.

\subsection{VLSI Fabrication}

To create a NEM switch logic library for VLSI-level implementation, the NEM switches have to be connected in circuits using metal interconnect technology. This can be achieved by using very large-scale heterogeneous integration of the NEM switches on top of high-density metal interconnect layers [22, 23]. Figure 3 shows a process for integrating the NEM switches on top of a Si wafer containing planarized metal interconnects. In this process, the Si device layer of a SOI wafer is transferred to a pre-fabricated Si wafer containing multi-layer metal interconnects. The layer transfer is done using wafer bonding with a thin intermediate polymer adhesive [24] as shown in Figure 3b. After wafer bonding, the SOI handle wafer and the buried oxide layer are sacrificially removed as depicted in Figure 3c. Thereafter, anchor structures are formed that mechanically and electrically connect the NEM switch layer

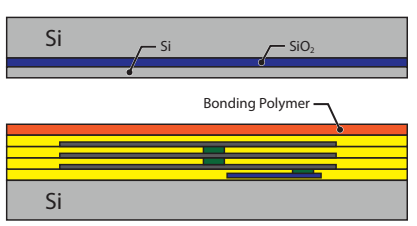

a)

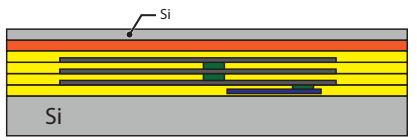

c)

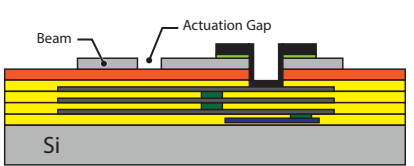

e)

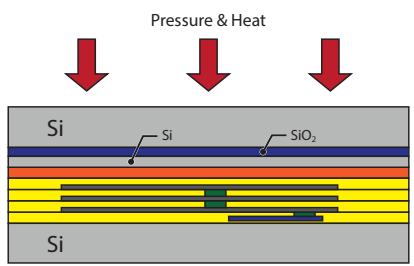

b)

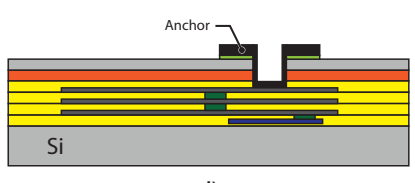

d)

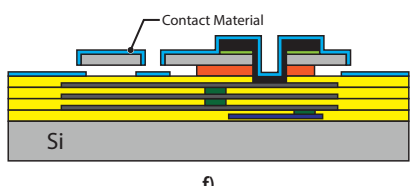

f)
Figure 3: Very large-scale heterogeneous integration of NEM switches on top of metal interconnect layers. (a), (b) Bonding a SOI wafer to a metal interconnect wafer using a thin intermediate polymer adhesive. (c) Removal of SOI handle wafer and buried oxide layer. (d) Formation of metal anchors. (e) Definition of NEM switches. (f) Free-etching of NEM switches and deposition of switch contact materials.

with the metal interconnects on the Si wafer as depicted in Figure 3d. Next, the outlines of the NEM switches are patterned in the transferred $\mathrm{Si}$ as shown in Figure 3e. Finally, the NEM switches are free-etched and the contact materials are deposited onto the switches as shown in Figure 3f, using a similar process as described in Section 2.2. The proposed heterogeneous integration technology is compatible with wafer-level processing and it is capable of implementing NEM switches with nanometer dimensions $[22,23]$.

\subsection{Standard Logic Cell Design}

The NEM switch device in this work is limited to 3 terminals, and thus lends itself to the complementary style of logic. Analogous to the p-type and n-type transistors in CMOS logic, a logic cell consists of a pull-up and pulldown network composed of NEM switches whose respective complementary pair is connected by a common gate as one of the inputs of the Boolean logic function. This style of logic differs from alternative topologies that can be obtained from 4-/5-/6-terminal devices [10, 13, 18, 19]. However, where we lose in terms of the capability to use more unique logic styles, we gain in terms of better scalability and optimization at the device level [4, 20]. Because our NEM switches are ambipolar, relying solely on the voltage difference between the gate and source (cantilever) for actuation, there is no need to create a p-type or n-type switch. A single switch design can be used for both the pull-up and pull-down networks, whose behavior depends on how the switch is biased at the source terminal (i.e., $V_{\mathrm{dd}}$ for pull-up, $V_{\mathrm{ss}}$ or GND for pull-down). In other 
words, a CMOS-like NAND gate can become a NOR gate by swapping $V_{\mathrm{dd}}$ and $V_{\mathrm{ss}}$ (or GND).

In the effort of bringing NEM switches to VLSI applications, a standard approach for designing logic cells was developed. Ultimately, we are limited in how close anchor electrode connections can be placed relative to the NEM switch device. The final size of the pitch chosen for the anchors is $4.5 \mu \mathrm{m}$, which can be arranged in a grid as shown in Figure 5a (circles). Interconnect wires can be routed along the horizontal and vertical channels shown on the grid and even beneath the anchors. This anchor pitch creates sufficient space to enable an interchangeable use of our soft and stiff NEM switches (Figure 4).

A single vertical column of 5 anchors is sufficient to create a simple inverter cell as one would place the pullup switch on the first 3 anchors, whereas the pull-down switch occupies the last 3 anchors. The middle anchor in a column of 5 is shared by the drain terminal of both switches. A more elaborate example is shown in Figure $5 \mathrm{~b}$, where the switches are placed on the anchor grid to form a NAND8 cell. Note how the pull-down switches connected in series share the same anchor from drain to source. The U-shaped path of the series switches makes it straightforward to connect the gate electrodes of the parallel switches in the pull-up network. Finally, connections are made through the available routing channels to complete the logic cell in a $45 \mu \mathrm{m} \times 27 \mu \mathrm{m}$ area as illustrated in Figure 5c. This relatively large area of $1215 \mathrm{\mu m}^{2}$ is due to the lithography limitation of the anchors, but assuming this can be improved through the miniaturization of anchor vias, we estimate that a NAND8 cell can fit into an area of $\sim 500 \mathrm{\mu m}^{2}$. This would put us on par with $350 \mathrm{~nm}$ CMOS technology, whose NAND8 cell can fit into a $480 \mathrm{\mu m}^{2}$ area. Based on the current state of our BEOL process, our fully-integrated NAND8 cell $\left(1215 \mu \mathrm{m}^{2}\right)$ is $\sim 2.5$ times larger than the $350 \mathrm{~nm}$ CMOS equivalent $\left(480 \mu^{2}\right)$. We have created a large variety of combinational cells from 2- to 16-input logic gates as well as sequential logic cells (flip-flops and latches) using the described standard approach.

Furthermore, because NEM switches have practically zero leakage, we can exploit this property to create dynamic circuits in which data is stored as charge on parasitic capacitances. In CMOS, such circuits must operate at a minimum clock rate so that data is read or refreshed before sufficient charge leaks away to change the logical state [25]. In the case of NEM switches, there is no need for a minimum clock rate. Figure 6 is an example of how one would implement a dynamic D flip-flop by exploiting the zero-leakage property of NEM switches. Both static and dynamic logic cells have been implemented in the development of the logic library.

Lastly, an advantage of the curved cantilever switch is the wide margin between $V_{\mathrm{pi}}$ and the breakdown voltage $[6,20,21]$. Measured breakdown voltages of curved cantilevers show that they are more than twice the pull-in voltage. This is particularly useful for creating a library

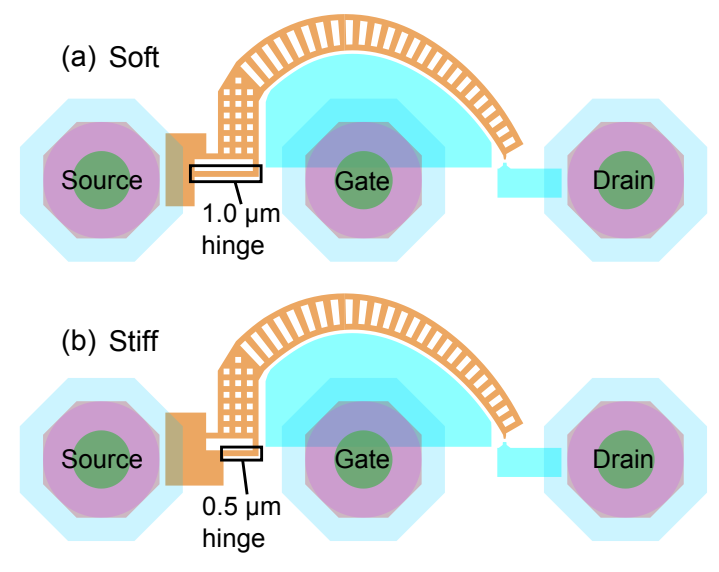

Figure 4: Layout of NEM switches with anchor electrode connections. The spacing of the anchors $(4.5 \mu \mathrm{m}$ pitch) allows seamless interchangeability between the two designs. The length of the hinge is an adjustable design parameter that influences the $V_{\text {pi }}$ of the switch. The soft switch design has a length of $1.0 \mu \mathrm{m}$, a $V_{\mathrm{pi}}$ of $7.6 \mathrm{~V}$, and a pull-in time of $48 \mathrm{~ns}$ (a). The stiff switch design has a length of $0.5 \mu \mathrm{m}$, a $V_{\mathrm{pi}}$ of $10.3 \mathrm{~V}$, and a pull-in time of $23 \mathrm{~ns}(\mathrm{~b}) . V_{\mathrm{pi}}$ and pull-in times are expected values obtained from finite-element analysis.

of buffering elements composed of NEM switches with different hinge lengths to fine tune the data and clocking paths for completing the timing closure of VLSI circuits [26]. The hinge length of the switch influences the switching speed as a stiffer switch results in a higher resonant frequency. At the same time, the stiffness also changes $V_{\mathrm{pi}}$ as the stiffest buffer in the library would have the highest $V_{\mathrm{pi}}$. This ultimately determines what the minimum operating voltage of the entire circuit should be, requiring the softer buffers in the circuit to be overdriven above their relatively lower $V_{\mathrm{pi}}$. But because of the wide margin between $V_{\text {pi }}$ and the breakdown voltage of the curved cantilever design, this is a design tuning technique that can be safely applied using our devices. Note that this particular technique directly manipulates the mechanical domain of the switching speed which is the dominant component of the overall delay when compared with the RC electrical delay of the device [27].

\subsection{Interface Measurement Circuits for NEM Switches}

The large parasitic loads of the measurement equipment can be problematic when trying to characterize NEM switches as the resulting surge currents from such loads can inadvertently weld the electrical contacts, ceasing further operation of the switch.

To overcome this difficulty, we added a single output switch that is actuated by the output of the circuit-undertest (CUT) so that the latter is decoupled from the measurement equipment. The source-drain voltage $\left(V_{\mathrm{sd}}\right)$ of the output switch is limited by means of an interfacing operational amplifier. The source terminal of the output switch is always set to a fixed voltage, typically $V_{\mathrm{dd}}$ or $V_{\mathrm{ss}}$, whereas the drain terminal is connected to the virtual ground of the operational amplifier. The amplifier uses an inverting topology that allows the virtual ground voltage 


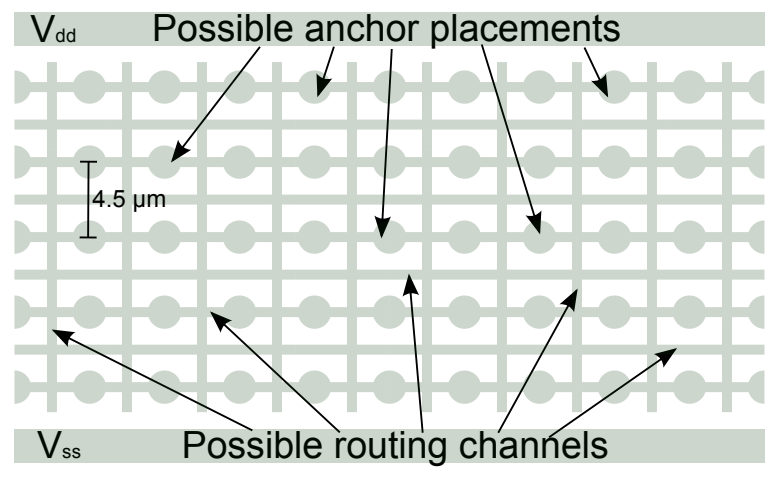

(a) Standard cell template with anchor grid and supply rails

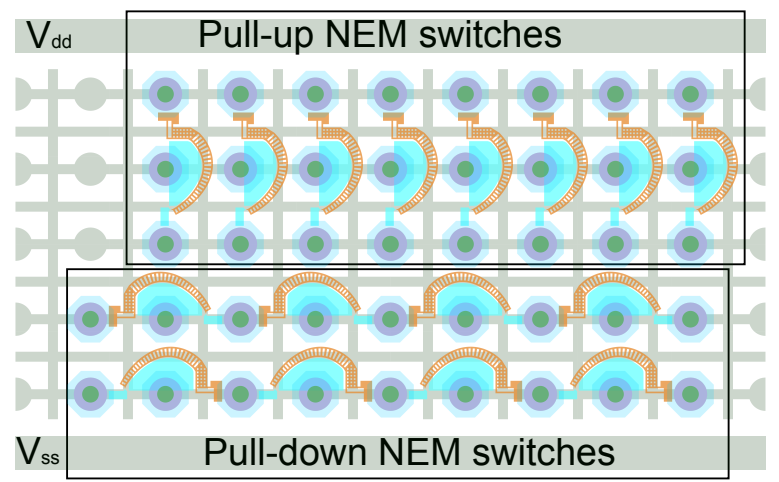

(b) NEM switches placed on anchor grid for a NAND8 cell

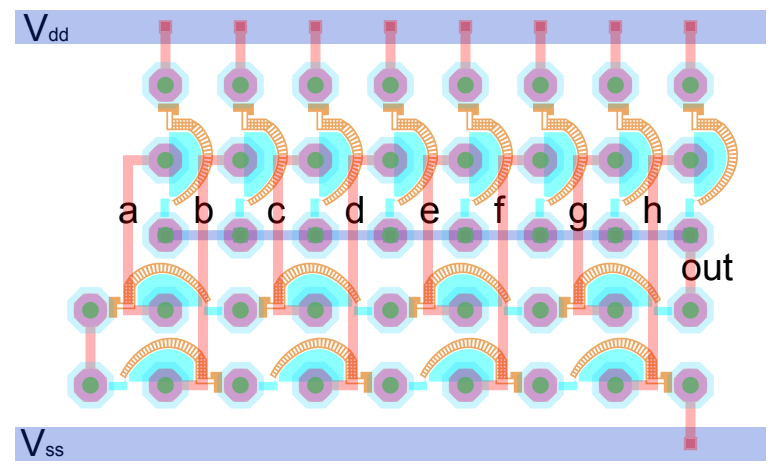

(c) Completed NAND8 cell with a $45 \mu \mathrm{m} \times 27 \mu \mathrm{m}$ footprint

Figure 5: Design approach for standard cell development using the full-integration process.

to be adjusted through the direct biasing of the positive input so that we can safely limit the voltage across the output switch while still operating the CUT at the full-voltage swing. The measured output is effectively the output of the inverting operational amplifier as it compensates relative to the virtual ground in response to the output switch driven by the CUT. Figure 7 shows the schematic of the interface circuit.

With this approach, we effectively limit the output voltage swing, but we also reduce the surge currents that otherwise may weld the nanoscale contact of the switch because of the large external capacitive load of the measurement setup [21]. We typically apply a $V_{\mathrm{sd}}$ of $2 \mathrm{~V}$ to $2.5 \mathrm{~V}$ across the output switch so that there is a suffi-

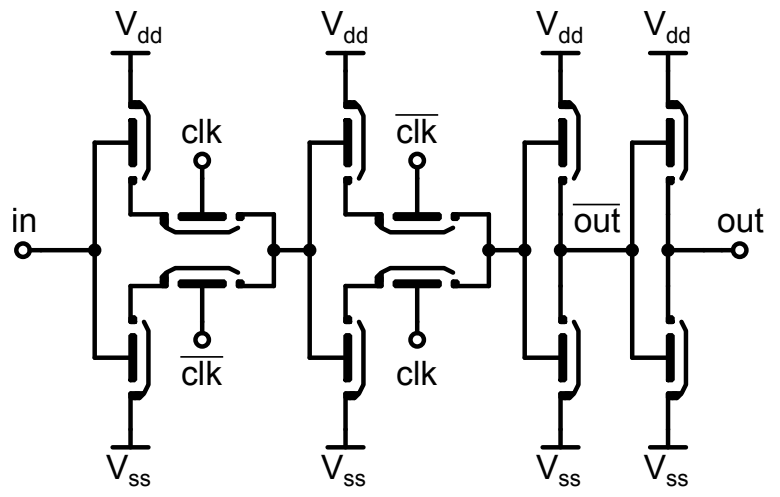

Figure 6: Schematic of a dynamic D flip-flop cell. As NEM switches have zero leakage, this unique property can be exploited to store data as charge on parasitic capacitances without the need of maintaining a minimum clock rate.

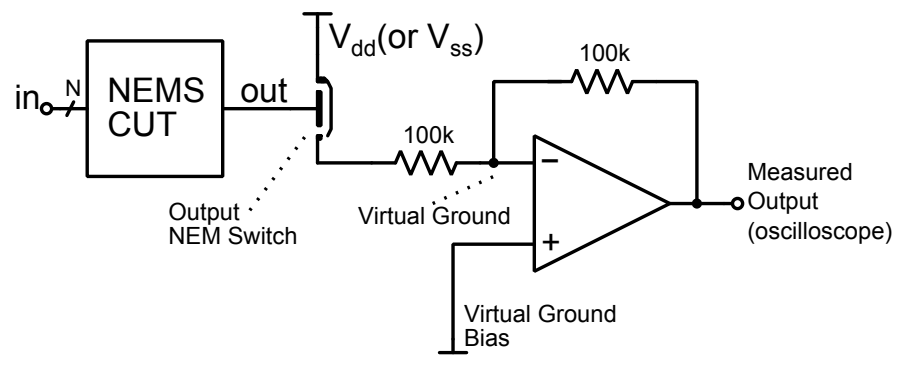

Figure 7: Schematic of the interface circuit for measurement using an output switch to sample the output of the CUT as well as to decouple it from the measurement equipment load. The potential across the output switch is kept at safe voltages through the use of the virtual ground of the operational amplifier.

ciently large voltage to allow the a-C to form conducting filaments. We have successfully measured prototype circuits with this measurement setup, as detailed in Section 4.

\subsection{Analytical Compact Model (ACM) Simulation}

Emerging electron devices need corresponding compact models in order to be brought seamlessly into the industrial design environments and the corresponding EDA tools. For this reason, we have developed an analytical compact model for the NEM switch.

As the functionality of the switch is based on the electrostatic actuation of a movable cantilever, the modeled behavior covers both the electrical and the mechanical regime. The development of the model is based on the hierarchical analysis of the device into separate modules. Each module is dedicated to a specific element of the switch (i.e., movable cantilever, contact) and targets either its electrical or its mechanical behavior. It is indispensable to have a balanced approach towards the modeling needs of both electrical and mechanical regimes, and this hierarchical modeling structure drives the model in this direction [28].

The classical parallel-plate capacitor approach lies at the core of the model, which is then enriched by scalable, empirical and physical formulations for describing various 


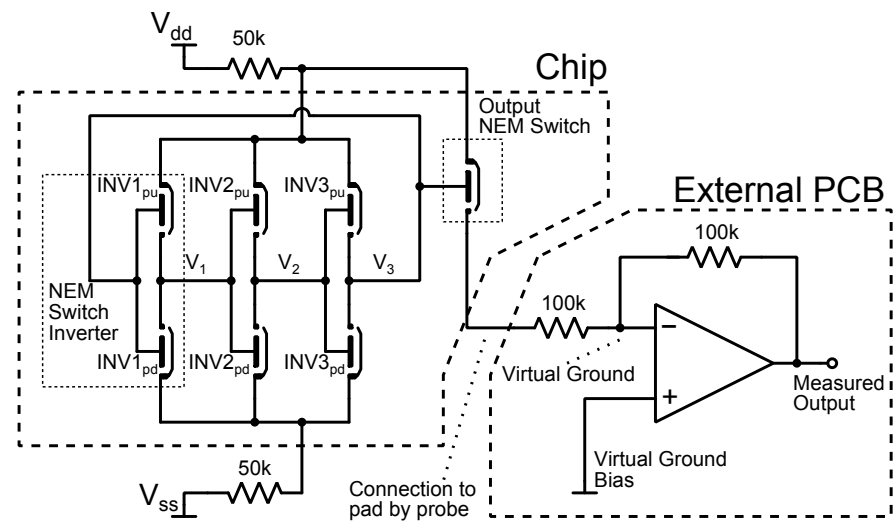

Figure 8: Schematic of the ring oscillator with output switch and measurement setup. $V_{\mathrm{dd}}$ and $V_{\mathrm{ss}}$ are in series with current-limiting resistors and are connected to the circuit through probe needles. A similar setup is also used for the standalone inverter and buffer with the addition of a function generator to drive the input of the respective CUT.

higher-order effects seen on real devices and their measurements. Overall, the model can be described as a robust set of equations that covers the complete electromechanical functionality of the switch. The Verilog-A language is a portable tool between circuit simulators that contains all the elements needed for this task, and thus it was used for the implementation of the model [29]. The model has been successfully validated against measurements and numerical simulations [28].

Compact models allow the emerging devices to be tested and validated at both the circuit- and system-level, and demonstrate their benefits and potential at a higher scale. Moreover, the analytical, physical and scalable characteristics of the compact model can be used to estimate the sensitivity and the trends of the behavior of the device on any physical and geometrical parameter, either as a single switch or at the circuit-level [30].

\section{Prototype Circuits and Measurements}

As a first experimental investigation of our NEM switch circuits, prototypes have been fabricated using only a single SOI wafer for fast turnaround and optimization of the devices. The fabrication steps for this development are a subset of the final steps of the full integration process. However, this limits connectivity to the same device layer for the time being, and therefore more complex logic circuits are not possible until the BEOL process is ready. The prototype circuits include a single-stage inverter, a buffer (two-stage inverter), and a three-stage ring oscillator. Because measurements were performed on circuits from different fabrication runs, there is some variability in the air gaps and in the quality of the conduction material, which can lead to different $V_{\mathrm{pi}}, R_{\mathrm{on}}$ and other device parameters.

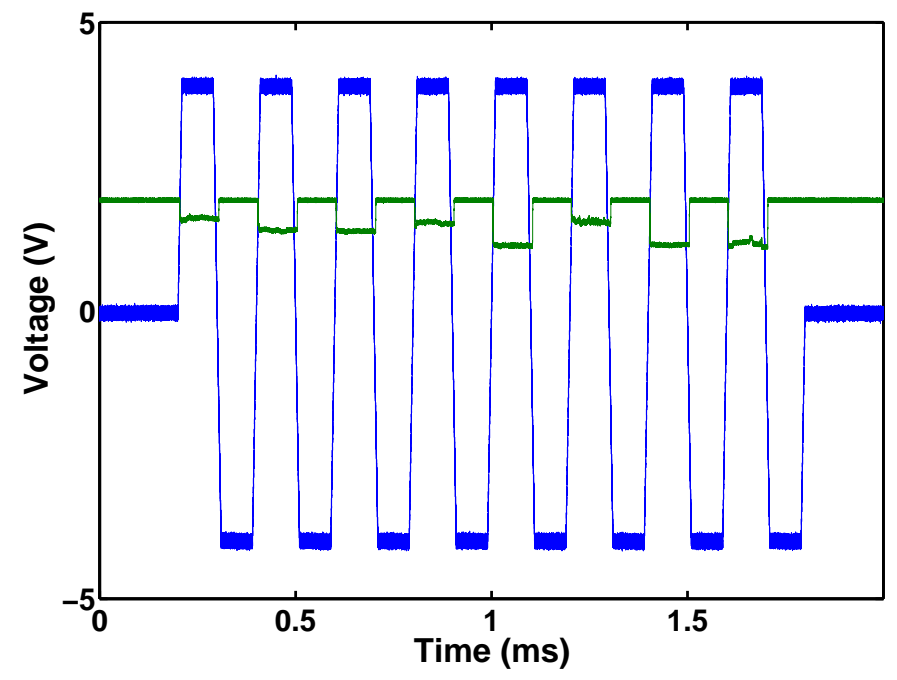

Figure 10: Output (green) of a standalone single-stage inverter driven by a $5 \mathrm{kHz} \pm 4 \mathrm{~V}$ input (blue). Measurement parameters: $V_{\mathrm{dd}}$ $=4 \mathrm{~V}, V_{\mathrm{ss}}=-4 \mathrm{~V}$, and $V_{\mathrm{sd}}=2 \mathrm{~V}$.

\subsection{Single-Stage Inverter}

The simplest logic circuit we can design is the singlestage inverter. It consists of two NEM switches in complementing configuration as shown as a subcomponent in Figure 8 and Figure 9a.

The soft switch design with a pull-in voltage of $7.6 \mathrm{~V}$ was used for this experiment, in which a $\pm 4 \mathrm{~V}$ input signal was applied to produce the measured output (Figure 10). The output trace is the result of three inversions: (1) NEM inverter (full rail-to-rail voltage swing internally), (2) output NEM switch, and (3) inverting amplifier (limited swing to control currents with interfacing measurement equipment and to observe resistance variations of the NEM switch). When the input signal is $V_{\mathrm{dd}}$, the output from the NEM inverter is $V_{\mathrm{ss}}$. This closes the output switch, causing the inverting amplifier to respond with a negative voltage with respect to the virtual ground to compensate for the increase in current from the output switch. When the input signal is $V_{\mathrm{ss}}$, the output from the NEM inverter is $V_{\mathrm{dd}}$. This opens the output switch and allows the inverting amplifier output to return to the virtual ground. As observed in Figure 10, the output exhibits slight differences in voltage each time the output switch closes. This is due to the active contact formation in the a-C layer, which alters the input resistance of the output amplification circuit and thus the inverting gain.

\subsection{Buffer (Two-Stage Inverter)}

Connecting two inverters in series creates a buffer circuit. By operating such a circuit, we can demonstrate how one logic gate can drive another logic gate under the fullvoltage swing. The measurement approach is similar to that of the single-stage inverter. The only small modifications to the measurement setup are that the source terminal of the output switch is now tied to $V_{\mathrm{ss}}$, and the virtual 


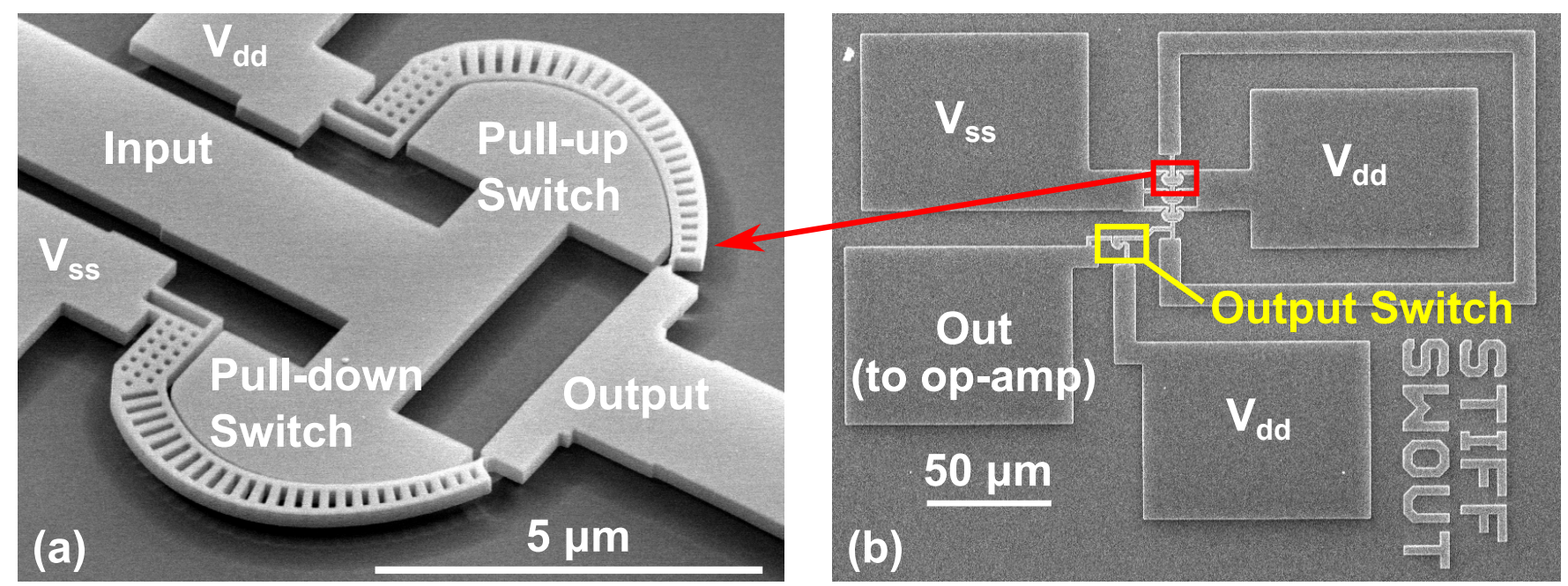

Figure 9: (a) SEM image of a single-stage NEM switch inverter and (b) SEM image of a 3-stage ring oscillator and output switch.

ground of operational amplifier is biased to a voltage close to $V_{\text {ss }}$. This will now cause the operational amplifier to respond with a voltage above virtual ground each time the output switch closes and then return to virtual ground when the output switch opens. The soft switch design was again used for this experiment, but on this particular fabrication run, the $V_{\mathrm{pi}}$ was lower than usual, namely in the range of $4 \mathrm{~V}$ to $5 \mathrm{~V}$ with air gaps of $25 \mathrm{~nm}$ to $30 \mathrm{~nm}$.

We first operated the circuit such that the $V_{\text {sd }}$ of the output switch is $2 \mathrm{~V}$ to $2.5 \mathrm{~V}$ to create a sufficient number of conduction filaments in the a-C contact and lower the $R_{\text {on }}$ so that we can see a reasonable output from the operational amplifier. Afterwards, we reduced $V_{\text {sd }}$ to $1 \mathrm{~V}$ and cycled the buffer in bursts of 1024 cycles. Figure 11 shows the buffer operating over the entire duration of the measurement.

\subsection{Three-Stage Ring Oscillator}

The ring oscillator consists of 3 inverter cells connected in series with the output of the final inverting stage connected to the input of the first inverting stage to create the self-oscillating feedback loop when the circuit is powered on (Figure 9b). This differs from NMOS-style singleswitch oscillators achieved in the past [18], which use a pull-up resistor instead of an additional, complementing switch.

To measure the ring oscillator, the $V_{\mathrm{dd}}$ and $V_{\mathrm{ss}}$ power supply rails are enabled for $100 \mu$ s to control the number of oscillations. The stiff switch with a simulated pull-in voltage of $10.3 \mathrm{~V}$ was used for this experiment. A $V_{\mathrm{dd}}$ and $V_{\text {ss }}$ of $\pm 7 \mathrm{~V}$ were needed to observe oscillations. While the NEM switches in the oscillator operate at the full railto-rail voltage swing of $14 \mathrm{~V}$, the output stage has a low bandwidth and thus limits the oscillations to $100 \mathrm{mV}$ (Figure 12a and Figure 12b). The frequency of the output was measured to be $6.7 \mathrm{MHz}$. In other runs of the same circuit design, these oscillations have consistently been in the $6 \mathrm{MHz}$ to $7 \mathrm{MHz}$ range, with an operating voltage of $13 \mathrm{~V}$ to $15 \mathrm{~V}$ (Figure 12c and Figure 12d). These oscillations have been sustained for as long as $50 \mu \mathrm{s}$. Similar to the previous measurements, the slight changes in the peak-topeak voltages result from the changing resistance of the a-C layer. As the propagation delay of NEM switches is mechanically dominated $[4,5,27]$, the resistive variations have a negligible effect on the operation speed and functionality of digital circuits.

After the oscillations have stopped, probing the power supply pads with a low voltage showed unconditional current flow from $V_{\mathrm{dd}}$ to $V_{\mathrm{ss}}$, which indicates a direct short circuit through welded switches in both the pull-up and pull-down network. Compared with hot-switching reliability experiments performed on standalone curved NEM switches [21], measurements in this case are done at larger voltages and with switches in a complementing configuration. This increases the possibility of a short circuit should one switch get temporarily stuck in the closed position and its complementing switch also close during this period of stiction. The resulting short-circuit current from the temporary stiction can ultimately lead to the welding of the nanoscale contacts, causing oscillations to cease. This fundamentally different experiment could explain the disparity in reliability when compared with single-switch experiments. Prevention of temporary stiction will require further investigation and optimization of the a-C contact material, perhaps by adjusting the thickness of the deposition such that nanoscale conducting filaments can still form but adhesion forces are kept sufficiently low to prevent stiction.

Furthermore, probing the output switch showed no unconditional current flow and thus no welding. This indicates the effectiveness of using an inverting operational amplifier as a protective readout circuit during measurement.

The ring oscillator is an excellent circuit to extract the propagation delay of a single inverter from measurements. A single cycle at the output corresponds to 2 passes of the 


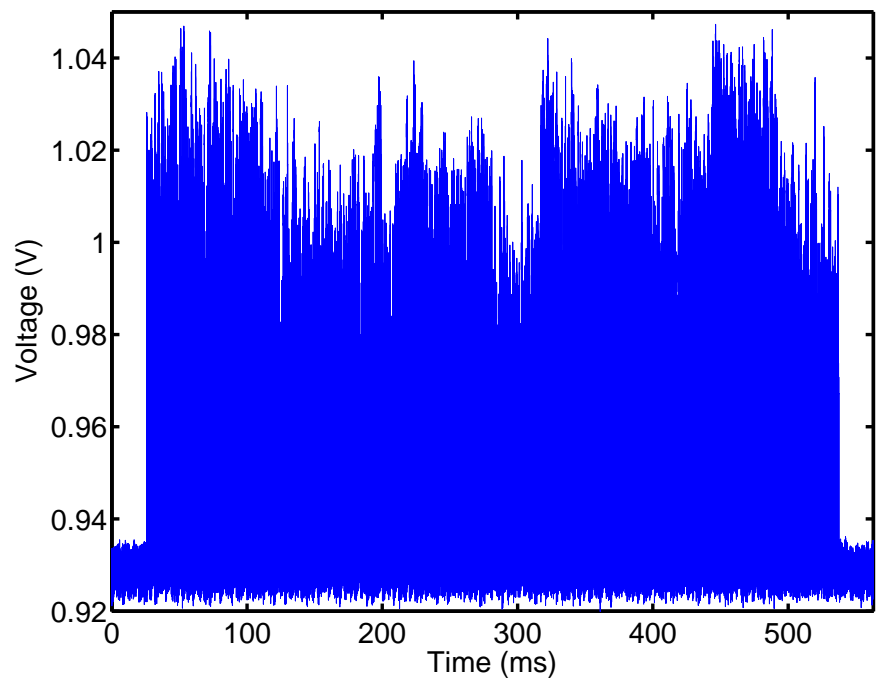

(a) Full-view of the 1024-cycle burst measurement of the buffer

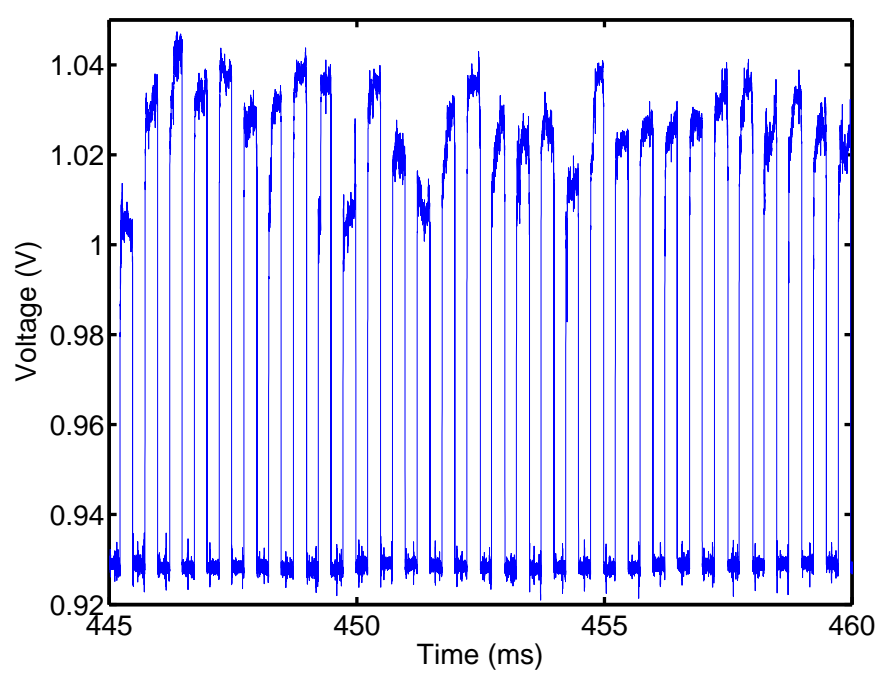

(b) A zoom-in on the burst measurement

Figure 11: Measured output of the NEM switch buffer actuated for 1024 cycles at $2 \mathrm{kHz}, V_{\mathrm{dd}}=5 \mathrm{~V}, V_{\mathrm{ss}}=0 \mathrm{~V}, V_{\mathrm{sd}}=1 \mathrm{~V}$. The variations in the amplitude reflect the changing contact resistance and are amplified by the output read-out circuit. Amplitude swings vary from $60 \mathrm{mV}$ to $120 \mathrm{mV}$ above the virtual ground.

oscillator or 6 inverter delays in total. With a measured cycle time of $148 \mathrm{~ns}(6.7 \mathrm{MHz}$ frequency), this results in a $25 \mathrm{~ns}$ propagation delay per inverter. Simulation results of a transient finite-element analysis of the tip-displacement of a single stiff switch as it makes contact (Figure 13a) and of a transient analysis of a single-stage inverter using the analytical compact model (Figure 13b) confirm the extracted delay [28, 30]. Furthermore, an equivalent schematic of Figure 8 was implemented in simulation to verify the functionality of the model and evaluate the consistency of the measured data. Ideal elements were used to describe the behavior of the operational amplifier and the readout circuitry of the setup. In addition, a modest parasitic capacitive load was added to the nodes in between the stages of the ring oscillator with a shunt connection to

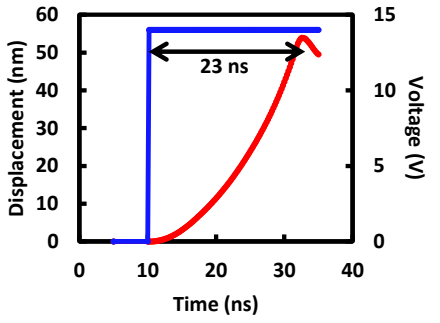

(a) Tip displacement

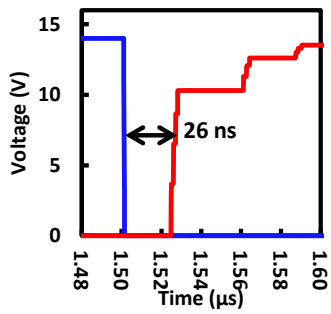

(b) Inverter delay
Figure 13: (a) Transient finite-element analysis of the actuation voltage rising to $14 \mathrm{~V}$ (blue) and the tip displacement (red) as the tip makes contact. (b) Analytical compact model simulation of propagation delay in a single-stage inverter measured from $50 \%$ of the input (blue) to $50 \%$ of the output (red). The step response is due to the modeled tip bouncing, but the first contact is sufficient to achieve $50 \%$ of the output swing.

the ground $\left(C_{\text {par }}=1 \mathrm{fF}\right)$. The stiffness of the cantilever was tuned to match $V_{\mathrm{pi}}$ obtained from the static characterization of stand-alone stiff NEM switches. The overall behavior of the system corresponds well to the measured data and results in an oscillating period of $152 \mathrm{~ns}$ (Figure 14).

\section{Conclusion}

We have fabricated and demonstrated the first NEM ring oscillator built with logical inverters using curved cantilever switches coated with a-C on the contact. The circuit uses a dedicated output stage to safely interface with the environment and operates at $6.7 \mathrm{MHz}$. The measured results match well with simulations, indicating that our stiff NEM switches have a switching time of $\sim 25 \mathrm{~ns}$. These demonstrated results are an important step towards developing scaled devices with a sub-micron length and an air gap of $5 \mathrm{~nm}$, enabling 1-V operation and sub-nanosecond propagation delays. The simplicity of the process flow ensures compatibility with present BEOL integration and paves a way for NEM-switch-based VLSI circuit design. The main challenge now is to optimize the a-C contact material. While it showed promise in operating under relatively low-voltage conditions, it still creates sufficiently strong adhesion forces to cause temporary stiction of the contact under high-voltage operation.

\section{Acknowledgement}

The authors gratefully acknowledge the support of Michel Despont and Ute Drechsler in the nanofabrication process, and Urs Duerig for fruitful discussions on the NEM switch device and characterization setup.

This work was supported by the European $7^{\text {th }}$ Framework Program (EU-FP7) under the grant agreement FP7NEMIAC (No 288670). 


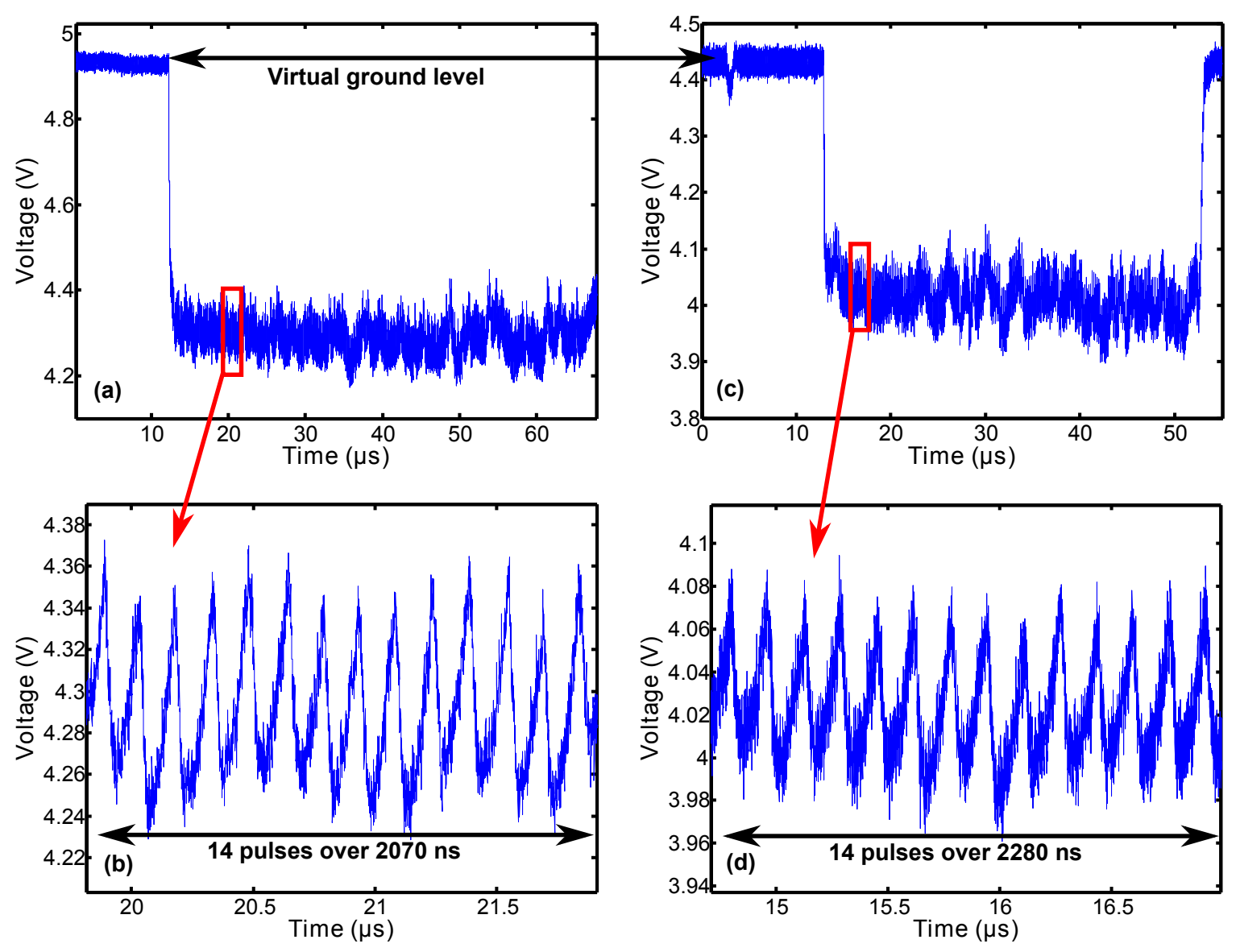

Figure 12: Measurements of the ring oscillator. While the oscillator operates at the rail-to-rail voltage swing internally, the limited bandwidth of the output stage attenuates the signal to avoid large surge currents that would fuse the contact. (a) Measured output of the ring oscillator with an operating voltage of $14 \mathrm{~V}$ and (b) a corresponding close-up showing $6.7 \mathrm{MHz}$ oscillations. A similar measured result of another sample with an operating voltage of $13 \mathrm{~V}$ is also shown in (c) and (d) oscillating at $6.1 \mathrm{MHz}$.

\section{References}

[1] International technology roadmap for semiconductors (2013). URL http://www.itrs.net

[2] D. Nikonov, I. Young, Overview of beyond-CMOS devices and a uniform methodology for their benchmarking, Proceedings of the IEEE 101 (12) (2013) 2498-2533.

[3] Y. Pu, Y. He, Z. Ye, S. Londono, A. Abbo, R. Kleihorst, H. Corporaal, From Xetal-II to Xetal-Pro: On the road toward an ultralow-energy and high-throughput SIMD processor, IEEE Transactions on Circuits and Systems for Video Technology 21 (4) (2011) 472 -484. doi:10.1109/TCSVT.2011.2125590.

[4] A. W. Knoll, D. Grogg, M. Despont, U. Duerig, Fundamental scaling properties of electro-mechanical switches, New Journal of Physics 14 (12) (2012) 84.

URL http://stacks . iop.org/1367-2630/14/i=12/a=123007

[5] O. Y. Loh, H. D. Espinosa, Nanoelectromechanical contact switches, Nature Nanotechnology 7 (5) (2012) 283-295. URL http://dx.doi.org/10.1038/nnano. 2012.40

[6] B. W. Soon, E. Ng, V. Hong, Y. Yang, C. H. Ahn, Y. Qian, T. Kenny, C. Lee, Fabrication and characterization of a vacuum encapsulated curved beam switch for harsh environment application, Journal of Microelectromechanical Systems 23 (5) (2014) 1121-1130.

[7] J. Fujiki, N. Xu, L. Hutin, I.-R. Chen, C. Qian, T.-J. K. Liu, Microelectromechanical relay and logic circuit design for zero crowbar current, IEEE Transactions on Electron Devices 61 (9) (2014) 3296-3302.

[8] B. Z. Tang, S. A. Bhave, R. Manohar, Low power asynchronous
VLSI with NEM relays, in: 2014 20th IEEE International Symposium on Asynchronous Circuits and Systems (ASYNC), 2014, pp. 85-92. doi:10.1109/ASYNC.2014.19.

[9] J. O. Lee, Y.-H. Song, M.-W. Kim, M.-H. Kang, J.-S. Oh, H.-H. Yang, J.-B. Yoon, A sub-1-volt nanoelectromechanical switching device, Nature Nanotechnology 8 (1) (2013) 36-40. URL http://dx.doi.org/10.1038/nnano.2012.208

[10] D. Lee, W. Lee, C. Chen, F. Fallah, J. Provine, S. Chong, J. Watkins, R. Howe, H.-S. Wong, S. Mitra, Combinational logic design using six-terminal NEM relays, IEEE Transactions on Computer-Aided Design of Integrated Circuits and Systems 32 (5) (2013) 653-666. doi:10.1109/TCAD.2012.2232707.

[11] I.-R. Chen, Y. Chen, L. Hutin, V. Pott, R. Nathanael, T.-J. K. Liu, Stable ruthenium-contact relay technology for low-power logic, in: 2013 Transducers Eurosensors XXVII: The 17th International Conference on Solid-State Sensors, Actuators and Microsystems (TRANSDUCERS EUROSENSORS XXVII), 2013, pp. 896-899. doi:10.1109/Transducers.2013.6626912.

[12] Y. Chen, R. Nathanael, J. Yaung, L. Hutin, T.-J. King Liu, Reliability of MEM relays for zero leakage logic, Proc. SPIE 8614 (2013) 861404-861404-7. doi:10.1117/12.2005719.

URL http://dx.doi.org/10.1117/12.2005719

[13] H. Fariborzi, F. Chen, R. Nathanael, I.-R. Chen, L. Hutin, R. Lee, T.-J. K. Liu, V. Stojanovic, Relays do not leak - CMOS does, in: 2013 50th ACM / EDAC / IEEE Design Automation Conference (DAC), 2013, pp. 1-4.

[14] H. Kam, Y. Chen, T.-J. K. Liu, Reliable microelectro-mechanical (MEM) switch design for ultra-lowpower logic, in: 2013 IEEE International Reliability 

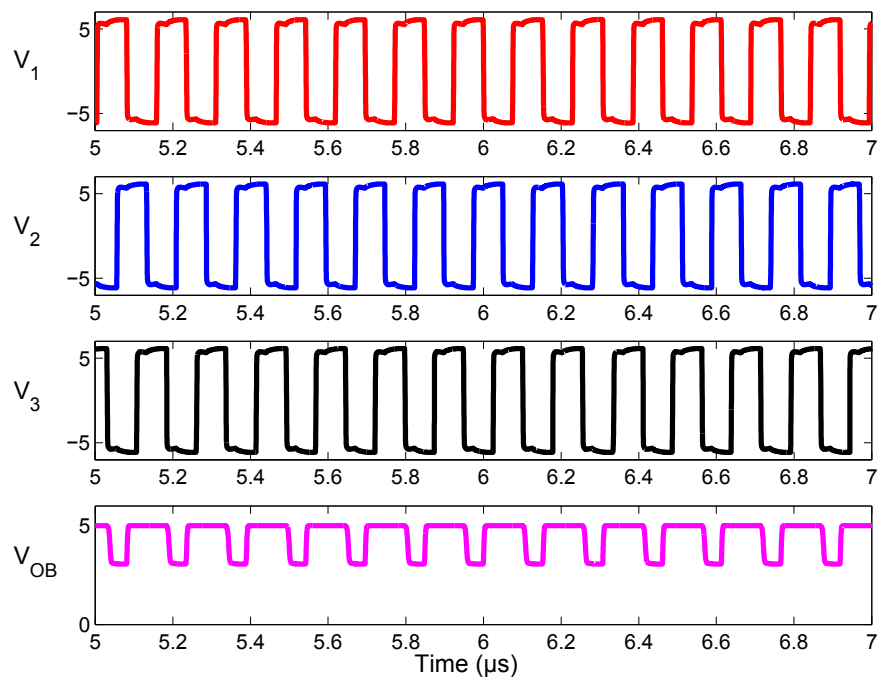

(a) Ring oscillator simulation waveform

Tip-Displacement (nm)
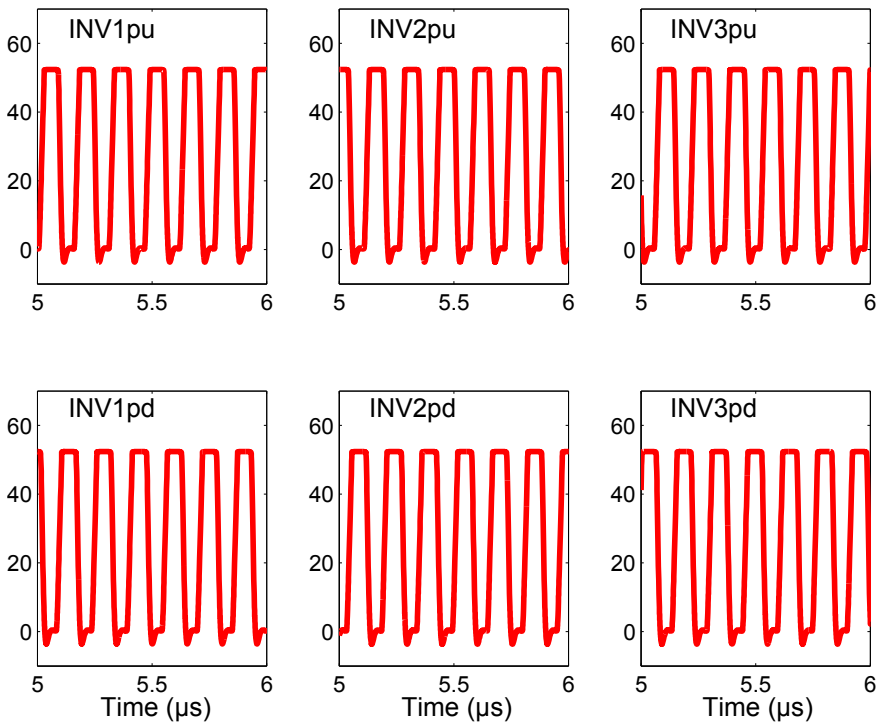

(b) Tip displacement of each NEM switch in nanometers

Figure 14: Simulation results of the measurement setup of the 3-stage ring oscillator using stiff switches based on the schematic in Figure 8. $V_{1}, V_{2}$ and $V_{3}$ are the inner signals of the oscillator from each inverter stage, whereas $V_{\mathrm{OB}}$ is the output received from the operational amplifier. $V_{\mathrm{dd}}$ is $7 \mathrm{~V}$ and $V_{\mathrm{ss}}$ is $-7 \mathrm{~V}$ as in the measurements. The simulated oscillation period ( $T=152 \mathrm{~ns}$ ) closely matches the measured results $(T=148 \mathrm{~ns})$. Ideal elements have been used for the output circuitry, with no parasitics, which allows a fast large-swing response in the simulation, in contrast to the measurement setup.

Physics Symposium (IRPS), 2013, pp. 6A.1.1-6A.1.5. doi:10.1109/IRPS.2013.6532044.

[15] T.-J. K. Liu, L. Hutin, I.-R. Chen, R. Nathanael, Y. Chen, M. Spencer, E. Alon, Recent progress and challenges for relay logic switch technology, in: IEEE Symposium on VLSI Technology, IEEE, Honolulu, Hawai, 2012, pp. 43-44.

[16] J. Munoz-Gamarra, N. Barniol, J. Juillard, Analysis of a MEMS-based ring oscillator, in: 2012 IEEE International Symposium on Circuits and Systems (ISCAS), 2012, pp. 2103-2106.

[17] J. Jeon, L. Hutin, R. Jevtic, N. Liu, Y. Chen, R. Nathanael, W. Kwon, M. Spencer, E. Alon, B. Nikolic, T.-J. K. Liu,
Multiple-input relay design for more compact implementation of digital logic circuits, IEEE Electron Device Letters 33 (2) (2012) 281-283

[18] M. Spencer, F. Chen, C. C. Wang, R. Nathanael, H. Fariborzi, A. Gupta, H. Kam, V. Pott, J. Jeon, T.-J. K. Liu, D. Markovic, E. Alon, V. Stojanovic, Demonstration of integrated micro-electro-mechanical relay circuits for VLSI applications, IEEE Journal of Solid-State Circuits 46 (1) (2011) 308320. doi:10.1109/JSSC.2010.2074370.

[19] H. Fariborzi, F. Chen, V. Stojanovic, R. Nathanael, J. Jeon, T.-J. K. Liu, Design and demonstration of microelectro-mechanical relay multipliers, in: Proc. IEEE Asian Solid State Circuits Conf. (A-SSCC), 2011, pp. 117-120. doi:10.1109/ASSCC.2011.6123618.

[20] D. Grogg, U. Drechsler, A. Knoll, U. Duerig, Y. Pu, C. Hagleitner, M. Despont, Curved in-plane electromechanical relay for low power logic applications, Journal of Micromechanics and Microengineering 23 (2013) 8 .

URL http://stacks.iop.org/0960-1317/23/i=2/a=025024

[21] D. Grogg, C. Ayala, U. Drechsler, A. Sebastian, W. Koelmans, S. Bleiker, M. Fernandez-Bolanos, C. Hagleitner, M. Despont, U. Duerig, Amorphous carbon active contact layer for reliable nanoelectromechanical switches, in: 2014 IEEE 27th International Conference on Micro Electro Mechanical Systems (MEMS), 2014, pp. 143-146.

[22] F. Zimmer, M. Lapisa, T. Bakke, M. Bring, G. Stemme, F. Niklaus, One-megapixel monocrystalline-silicon micromirror array on CMOS driving electronics manufactured with very large-scale heterogeneous integration, Journal of Microelectromechanical Systems 20 (3) (2011) 564-572.

[23] M. Lapisa, G. Stemme, F. Niklaus, Wafer-level heterogeneous integration for MOEMS, MEMS, and NEMS, IEEE Journal of Selected Topics in Quantum Electronics 17 (3) (2011) 629-644.

[24] F. Forsberg, A. Lapadatu, G. Kittilsland, S. Martinsen, N. Roxhed, A. Fischer, G. Stemme, B. Samel, P. Ericsson, N. Hoivik, T. Bakke, M. Bring, T. Kvisteroy, A. Ror, F. Niklaus, CMOSintegrated $\mathrm{Si} / \mathrm{SiGe}$ quantum-well infrared microbolometer focal plane arrays manufactured with very large-scale heterogeneous 3-d integration, IEEE Journal of Selected Topics in Quantum Electronics 21 (4) (2015) 1-11.

[25] S.-M. Kang, Y. Leblebici, Dynamic logic circuits, in: CMOS Digital Integrated Circuits: Analysis and Design, 3rd Edition, McGraw-Hill, 2003, Ch. 9.

[26] A. B. Kahng, J. Lienig, I. L. Markov, J. Hu, Timing closure, in: VLSI Physical Design: From Graph Partitioning to Timing Closure, Springer, 2011.

[27] S. Rana, Q. Tian, A. Bazigos, D. Grogg, M. Despont, C. Ayala, C. Hagleitner, A. Ionescu, R. Canegallo, D. Pamunuwa, Energy and latency optimization in NEM relay-based digital circuits, IEEE Transactions on Circuits and Systems I: Regular Papers 61 (8) (2014) 2348-2359.

[28] A. Bazigos, C. Ayala, M. Fernandez-Bolanos, Y. Pu, D. Grogg, C. Hagleitner, S. Rana, T. Qin, D. Pamunuwa, A. Ionescu, Analytical compact model in Verilog-A for electrostatically actuated ohmic switches, IEEE Transactions on Electron Devices 61 (6) (2014) 2186-2194.

[29] M.-A. Chalkiadaki, C. Valla, F. Poullet, M. Bucher, Why- and how- to integrate Verilog-A compact models in SPICE simulators, Int. J. Circ. Theor. Appl. 41 (11) (2013) 1203-1211. URL http://dx.doi.org/10.1002/cta.1833

[30] A. Bazigos, C. L. Ayala, S. Rana, D. Grogg, M. FernandezBolaos, C. Hagleitner, T. Qin, D. Pamunuwa, A. M. Ionescu, Electromechanical design space exploration for electrostatically actuated ohmic switches using extended parallel plate compact model, Solid-State Electronics 99 (0) (2014) 93 - 100. doi:http://dx.doi.org/10.1016/j.sse.2014.06.030.

URL http://www.sciencedirect.com/science/article/pii/ S0038110114001634 